\title{
An Evaluation of Graph Algorithms for the Wind Farm Cable Layout Problem under Electrical Aspects
}

\author{
Sascha Gritzbach \\ Institute of Theoretical Informatics \\ Karlsruhe Institute of Technology \\ Karlsruhe, Germany \\ sascha.gritzbach@kit.edu
}

Torsten Ueckerdt

Institute of Theoretical Informatics

Karlsruhe Institute of Technology

Karlsruhe, Germany

torsten.ueckerdt@kit.edu

\author{
Hüseyin Çakmak \\ Institute for Automation and Applied Informatics \\ Karlsruhe Institute of Technology \\ Karlsruhe, Germany \\ hueseyin.cakmak@kit.edu
}

\author{
Pascal Mehnert \\ Department of Informatics \\ Karlsruhe Institute of Technology \\ Karlsruhe, Germany
}

\author{
Veit Hagenmeyer \\ Institute for Automation and Applied Informatics \\ Karlsruhe Institute of Technology \\ Karlsruhe, Germany \\ veit.hagenmeyer@kit.edu
}

\begin{abstract}
The task of the Wind Farm Cable Layout Problem is to design a cable system between turbines and substations such that all turbine output can be transmitted to the substations. This problem can be modelled with different levels of complexity. While a higher level of complexity yields solutions that can be implemented in a real-world setting more readily, problem instances also become more difficult to solve or even remain intractable. More simplistic models are easier to solve but their usability could be inhibited. One such more simplistic model for installation cost minimization contains a network flow and a suitable minimum-cost flow algorithm provides good cable layouts on instances with up to 500 turbines within tens of seconds. The question remains whether those cable layouts are feasible for electrical flows as well.

We propose a workflow to evaluate cable layouts generated from such algorithms with respect to their performance under electrical aspects. This workflow converts the output of cable layout optimization algorithms to power flow models. The power flow models are simulated using the simulation framework eASiMOV.

The evaluation of the power flow simulations under electrical metrics shows that output from the minimum-cost flow algorithm and from an approach solving a Mixed-Integer Linear Program perform very well under electrical aspects on a vast majority of input instances. For the remaining minority we are able to identify structures in the solutions that result in a worse performance. These observations can be used by the algorithm engineers as possible directions for future improvements.
\end{abstract}

Index Terms-Wind Farm Cable Layout, Graph Algorithm, Network Flow, Power Flow Analysis

\section{INTRODUCTION}

Harvesting wind energy is a mature and comparatively cheap way of providing clean energy. Hence, it plays an important role in limiting the effects of climate change. The European Union has recently revealed plans to increase the installed capacity of offshore wind energy to $60 \mathrm{GW}$ by 2030 and to $300 \mathrm{GW}$ by 2050 [1].
In an offshore wind farm, electrical energy is generated by a number of wind turbine generators. The production of all wind turbines is gathered via the internal cabling of the wind farm and transported to one of potentially multiple offshore substations. All substations are connected to an onshore grid in order to make the electrical energy generated by the wind farm available to the rest of the power grid.

Designing the topology of the internal cabling is an important step in the process of planning a wind farm. Installation costs for the internal cabling account for roughly $5 \%$ of the total installation cost [2]. Technical aspects such as line losses contribute to the lifetime profitability of a wind farm.

\section{A. Related Work}

One way to design the internal cabling is to choose one of several existing design patterns. With a power flow analysis, different design patterns can be compared under technical aspects, such as power losses, voltage levels, and redundancy [3]. The cable topology planner can then apply the (for their priorities) best pattern. Alternatively, a broad variety of mathematical optimization approaches, such as MixedInteger Linear Programming (MILP), or metaheuristics, such as Simulated-Annealing, allow optimizing the cable layout with respect to a target function under certain features. Target function optimization could be installation cost minimization or power loss minimization. Examples for features are the choice between Direct and Alternating Current, redundancy requirements, incorporating wind stochasticity or freedom of choice from several available cable types. For a survey on such optimization techniques, see [4].

The design of the internal cabling can also be modelled as a graph-theoretic problem [5]. This opens up the mathematicians' and algorithmicians' toolboxes even further and 


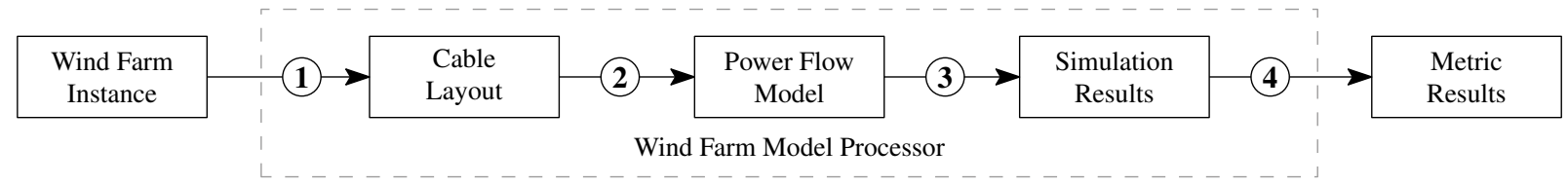

Fig. 1. Overview of intermediate steps within the Wind Farm Model Processor

promises new solution methods. The authors of [5] propose a hierarchical decomposition of the Wind Farm Cable Layout Problem. For the case of only one available cable type, this decomposition leads to well-known graph problems such as the Minimum Spanning Tree Problem. If the wind farm planner can use multiple cable types within the same wind farm, the authors are able to solve the Circuit Problem, i. e., a set of turbines must be connected but only one turbine has a direct connection to the single substation, optimally for at most 14 turbines. Bigger instances and more complex settings, such as multiple substations, remain intractable - due to exponentially growing running time or increasing complexity of the problem.

Similarly, the cable topology optimization can be modelled as a minimum-cost flow problem with a step-cost function that represents multiple cable types. We refer to this variant of the Wind Farm Cable Layout Problem as WCP. WCP can be solved by means of graph algorithms [6]-[8]. The graph algorithm used there is called Negative Cycle Canceling (NCC) It optimizes the cable layout with respect to installation cost minimization. This algorithm computes cheap, albeit not costminimal, solutions for wind farms of up to 500 turbines within 1.5 minutes. In the same papers, the cable layouts from the NCC algorithm are compared to a MILP formulation [9] solved by Gurobi [10] with a maximum running time of one week. Both approaches yield comparably priced cable topologies that are feasible within the underlying network flow model. However, no consideration is given to the electrical aspects of the solutions: Are the cable layouts feasible under an electrical flow model? Are they sensible under electrical metrics such as power losses or voltage stability?

\section{B. Contribution and Outline}

The main contribution of this work is a new coupling of graph algorithms for WCP and power system analysis with two goals: Assess the quality of graph algorithm solutions for realworld usage and provide feedback to algorithm engineers. A work flow for investigating the electrical properties of the output of the NCC algorithm and the MILP is proposed (Sec. II). This includes a description of the power flow models (Sec. II-A) and a methodology to obtain the required electrical parameters from abstract input parameters (Sec. II-B). The work flow is used to compare the electrical properties of the algorithm output to real wind farm data (Sec. III). We show that the computed cable layouts perform well under electrical flows, as long as meshes are absent, substations are not connected via the internal cabling, and installed cables are not disproportionately long. Those observations yield recommendations for further algorithmic work (Sec. IV).

\section{Methodology}

An overview of the proposed work flow is shown in Fig. 1. The work flow invokes the NCC algorithm or an MILP [7], [8] to compute an optimized cable layout (Fig. 1, Step 1) or it uses a precomputed cable layout in GraphML-format [11]. In Step 2, the cable layout is converted into a power flow model that can be processed by the eASiMOV (Energy Systems Analysis, Simulation, Modeling, Optimization and Visualization) software framework [12], [13]. This framework uses the open-source simulation package MATPOWER [14] to simulate the power flow models (Step 3). In Step 4, a range of metrics are obtained from the simulation results.

While there are more powerful models to simulate power systems, we believe the power flow model is a most suitable link between the complexity of power systems in real-world wind farms and the simplifying network flow model in WCP.

The whole work flow is realized by the novel Wind Farm Model Processor, programmed in Python 3. Multiple input instances can be processed in a single invocation. A commandline interface (CLI) provides fine-granulated control over all processing steps and facilitates extensions to all functionality that is provided in the Python-APIs of all external software (or the CLI provided by the C++-code for the NCC algorithm).

\section{A. Power Flow Models}

This section states the technical parameters required in the setup of the Wind Farm Model Processor. Fig. 2 shows a wind farm as a power flow model with all electrical components.

Turbines are modelled as a generator that is connected via a Low-Voltage (LV) generator $(P V)$ bus and a step-up transformer to a Medium-Voltage (MV) load $(P Q)$ bus. For the generator, the rated power and active power injection must be specified. The voltages at the buses correspond to the operating voltages of the generator and the internal cabling, respectively. Substations are modelled as an MV load (PQ) bus connected to the internal cabling, a transformer and a HighVoltage (HV) slack bus. The voltage of the MV bus coincides with the voltage of the internal cabling and the voltage of the HV buses can be set independently. The transformers in turbines and substations are defined by rated power, short-circuit impedance, and $X / R$ ratio. Note that the HV slack buses can also be replaced by load buses connected via external cables to a single common HV slack bus with an external grid. Internal cables are modelled by a single $\pi$ transmission line each that connect two MV buses. For the cables, resistance, reactance, and capacitance must be specified, all of which can be set as a per-unit-of-length value, as well as rated apparent power and the length of the line. 


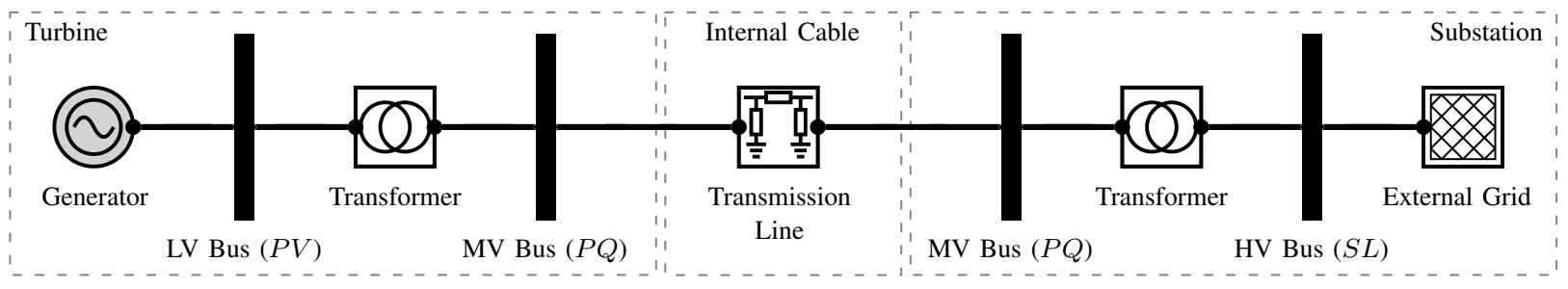

Fig. 2. Schematic representation of the components of a power flow model for wind farms

\section{B. Power Flow Models from NCC and MILP output}

For the evaluation of the NCC and MILP output, the instances are transformed into power flow models, in which the parameters must be set as defined in Sec. II-A.

The cables types [5] used in the evaluation of the optimization algorithms [7], [8] are given by thermal capacities (again in turbine production units) and costs per unit of length as shown in Table I. They were used for a proposed wind farm operating with an internal cabling at a voltage of $33 \mathrm{kV}$ [5]. The electrical parameters of the cables in the power flow models can be specified as follows: Take, for example, a selection of 3-core, XLPE-insulated submarine cables manufactured by Nexans [15] with different crosssectional areas and their rated currents as seen in Table II. By means of (1) the maximum apparent power per turbine $S_{\text {Trb }}$ can be computed for any particular cable type if it were used at their full capacity cap according to Table I.

$$
S_{\mathrm{Trb}}=\sqrt{3} \cdot 33 \mathrm{kV} \cdot \text { RatedCurrent } / \text { cap }
$$

This allows us to set the rated power at the generator buses, whereas WCP only assumes a per-unit output from turbines. While nowadays bigger turbines are used, the available cable types do not allow turbines with higher ratings than $3 \mathrm{MW}$, which we choose as the power rating of the generators. With the apparent power per turbine computed from the biggest cable type, this yields sufficient capacity for a plausible power factor of 0.92 . For each of the four cable type capacities, we choose the Nexans cable of smallest cross-sectional area that allow $3 \mathrm{MW}$ generators. The source for the corresponding values for resistance, reactance, and capacitance is [15].

As a wind turbine generator with $3 \mathrm{MW}$ we use an offshore version of the V112 by Vestas, which has a rotor diameter of $112 \mathrm{~m}$. We use $33 \mathrm{kV} / 690 \mathrm{~V}$ step-up transformers with a rated apparent power of $3.45 \mathrm{MVA}$, i.e., 1.15 times the rated active power of the wind turbine generator. From an example offshore wind farm in DIgSILENT PowerFactory [16] we obtain a short-circuit impedance of the transformer of $6 \%$ with an $X / R$ ratio of roughly $12: 1$.

For the offshore substation, a typical transformer with a primary voltage of $230 \mathrm{kV}$ and a short-circuit impedance of $3 \%$ is used. The impedance of the transformer was assumed to be purely reactive. Contrary to transformers at the turbines, the rated apparent power must be infered from the capacity of the substation $\left(\mathrm{cap}_{\mathrm{sub}}\right)$, which is given by the input instances. The rated apparent power of a substation is calculated as
TABLE I

CABLE TYPES FROM [5] USED IN THE EVALUATION OF NCC AND MILP

\begin{tabular}{lrrcc}
\hline Cable Type & $\mathrm{a}$ & $\mathrm{b}$ & $\mathrm{c}$ & $\mathrm{d}$ \\
\hline Capacity & 5 & 8 & 12 & 15 \\
Cost & 20 & 25 & 27 & 41 \\
\hline
\end{tabular}

TABLE II

APPARENT POWER PER TURBINE FOR EACH CABLE TYPE AND CONDUCTOR SIZE

\begin{tabular}{cccccc}
\hline $\begin{array}{c}\text { Cross-Section } \\
\left(\mathrm{mm}^{2}\right)\end{array}$ & $\begin{array}{c}\text { Rated Current } \\
\text { (A) }\end{array}$ & \multicolumn{5}{c}{$\begin{array}{c}\text { Apparent } \\
\text { (AVA) }\end{array}$} \\
& & 5 & 8 & 12 & 15 \\
\hline $\mathbf{9 5}$ & 291 & $\mathbf{3 . 3 3}$ & 2.08 & 1.39 & 1.11 \\
120 & 330 & 3.77 & 2.36 & 1.57 & 1.26 \\
185 & 411 & 4.70 & 2.94 & 1.96 & 1.57 \\
$\mathbf{2 4 0}$ & 470 & 5.37 & $\mathbf{3 . 3 6}$ & 2.24 & 1.79 \\
400 & 627 & 7.17 & 4.48 & 2.99 & 2.39 \\
$\mathbf{5 0 0}$ & 699 & 7.99 & 4.99 & $\mathbf{3 . 3 3}$ & 2.66 \\
630 & 777 & 8.88 & 5.55 & 3.70 & 2.96 \\
$\mathbf{8 0 0}$ & 852 & 9.74 & 6.09 & 4.06 & $\mathbf{3 . 2 5}$ \\
\hline
\end{tabular}

$S_{\mathrm{sub}}=\operatorname{cap}_{\mathrm{Sub}} \cdot P_{\mathrm{Trb}} \cdot(\cos \phi)^{-1}$ where $P_{\mathrm{Trb}}$ is the rated power of a wind turbine generator and an assumed power factor of $\cos \phi=(1.15)^{-1} \approx 0.87$.

The length of all transmission lines is computed from the input instances, which only specify relative positions on an arbitrary scale, by a scaling factor such that the minimum turbine distance is $700 \mathrm{~m}$, i. e., 6.25 times the rotor diameter.

\section{Evaluation}

All analyses in this section use the cable layouts of the 1000 synthetic instances (originally from [9]) randomly selected for the comparison of the MILP and the NCC algorithm with and without neighborhood heuristics in $[8$, Sec. 6.2]. These wind farms consist of up to 500 turbines. In the following, any reference to the NCC algorithm means the NCC algorithm without neighborhood heuristics. These cable layouts are evaluated with respect to average and maximum line loading, active power losses, reactive power injection, and voltage stability.

\section{A. Line loading}

In this part, the usage of the different cable types and the line loading is investigated. The loading of a transmission line is defined as the ratio of current flowing through that line according to the power flow simulation and its rated current. 
TABLE III

NUMBER OF CABLES USING EACH CABLE TYPE AVERAGED OVER ALL 1000 CABLE LAYOUTS OBTAINED FROM NCC AND MILP

\begin{tabular}{llccc}
\hline Cable Type & $(5,20)$ & $(8,25)$ & $(12,27)$ & $(15,41)$ \\
\hline NCC & 114.1 & 5.10 & 5.42 & 0.077 \\
MILP & 114 & 4.93 & 5.68 & 0.033 \\
\hline
\end{tabular}

Table III shows the average number of times each cable type is used across all 1000 instances. For both approaches, a vast majority of cables is chosen from the smallest cable type. This is not surprising as all turbines need to be connected and a capacity of 5 is sufficient to collect large parts of the outer areas of the wind farms. Both algorithms also use the bigger cable types; the NCC algorithm slightly more than the MILP.

The average line loadings across all instances are between $34 \%$ and $62 \%$ for the MILP and between $18 \%$ and $61 \%$ for NCC, with about $98 \%$ of all cables between $40 \%$ and $60 \%$. These small percentages arguably arise from a big amount of cables from the smallest type which connect the outer turbines. The mean average line loading across all instances is 0.58 percentage points smaller for the NCC algorithm than for the MILP. The difference seems neglectibly small, in particular since for the NCC, the instance with $18 \%$ average line loading is a single outlier. In this instance, no optimization took place as addressed in [8]. An overwhelming majority of instances (934 for NCC and 966 for MILP) show a maximum line loading between $90 \%$ and $91 \%$. In these cases, at least one cable is fully saturated to its capacity in the network flow. Twelve instances for NCC and eleven instances for MILP have a smaller maximum loading. The maximum loading of eleven cable layouts from NCC and four from MILP exceeds $95 \%$ (maximum values of $153 \%$ and $107 \%$ ). Those extremely high loadings are worrisome. Further inspection of those cable layouts reveals that all share one of two properties: The cable layout includes a cycle, i.e., one turbine has two paths to the same substation, or a turbine has paths to different substations. In both scenarios, there is a turbine where the incoming flow is split up. With network flow, any split is allowed. This does not hold for electrical flow. Thus, electrical flow and network flow can take different values. This can result in the observed overloads. Those scenarios are included in the recommentation to the algorithm engineers in Sec. IV.

Fig. 3 shows an example of an overload in a cycle. On each transmission line, a tuple states the absolute loading in the network flow model and the relative loading in the power flow model. From turbine T2, 20 units of flow are split up between the connection to turbine T3 (five units on a cable with a capacity of 5) and the connection to the substation (15 units on a cable with a capacity of 15). In the power flow model, this split is not maintained. Instead, more current flows on the substation line. This causes an overload, while the network flow respects all cable capacities.

Nonetheless, for a vast majority of instances both algorithms yield good cable layouts with respect to line loading.

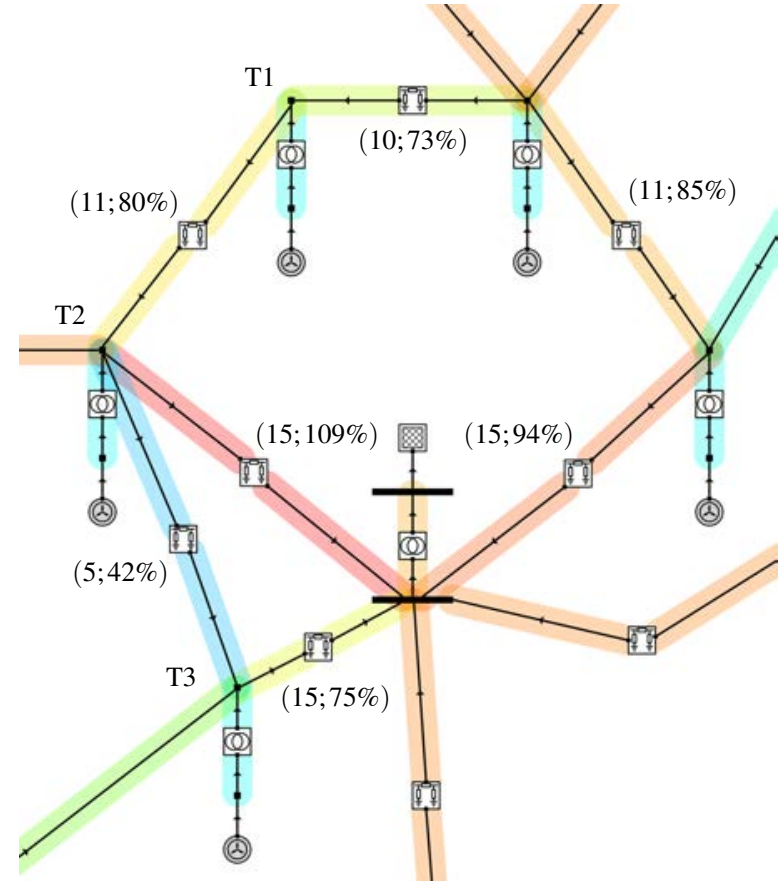

Fig. 3. A cycle in a power flow model causes an overload. Annotations to lines show the absolute loading in the network flow model in units of flow and the line loading in the power flow model as percentage values. The latter is also color-coded. The arrows indicate the direction of active power flow.

\section{B. Active Power Losses}

In this section, the active power losses along the transmission lines are investigated. As the absolute losses of the transmission lines we consider the difference between the active power injected by the transformers in the turbines into the internal cabling and the active power received at the external grid in the substations. To ensure comparability across all instances, we use ratios: absolute losses divided by turbine transformer active power output. Notably, losses inside the transformers are not considered. The losses at the turbines do not depend on the layout but are a constant depending on the parameters of generators and transformers. The impedance of transformers in substations was set as purely reactive (cf. Sec. II-B).

Fig. 4 shows the losses in the cable layouts computed by the NCC algorithm and the MILP. For both approaches, the losses are computed for all 1000 instances and sorted increasingly. NCC and MILP produce very similar results with a mean difference of only 0.0033 percentage points in favor of NCC. The horizontal line at $y=0.548$ shows a reference value from the literature for losses in a case study on an offshore wind farm with 95 turbines at a rated power of $4 \mathrm{MW}$ with a tree-like cable layout [17]. In comparison, NCC and MILP provide cable layouts with fewer losses on approximately $77 \%$ of all instances. Both algorithms yield a loss ratio of less than 0.75 on $97 \%$ of all instances. The maximum loss ratios are $0.98 \%$ for both MILP and NCC.

Thus, the optimization algorithms perform similarly with respect to active power losses and show a reasonable performance compared to a case study from the literature. 


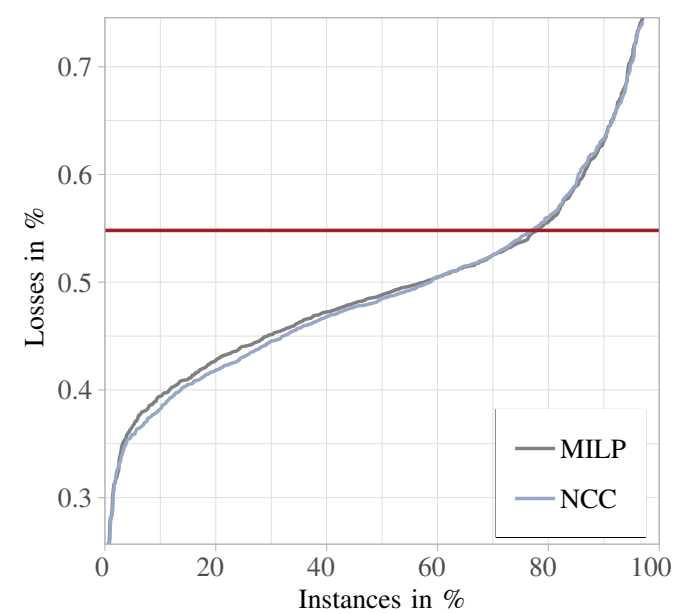

Fig. 4. Comparison of total losses in NCC and MILP generated cable layouts. For each algorithm, the loss ratios of all instances are sorted ascendingly. Loss ratios are computed as the difference between active power injected at transformers in turbines and active power received at external grids in substations, normalized by the total active power injection at transformers in turbines. The horizontal line gives a possible reference value of $0.548 \%$ [17].

TABLE IV

DISTRIBUTION OF POWER FACTORS AT SUBSTATIONS PER INSTANCE

\begin{tabular}{llcccc}
\hline Quantiles & max & median & $75 \%$ & $90 \%$ & $\min$ \\
\hline \multirow{7}{*}{ NeC } & 0.9951 & 0.9879 & 0.9872 & 0.9862 & 0.9734 \\
MILP & 0.9927 & 0.9879 & 0.9871 & 0.9864 & 0.9840 \\
\hline \multicolumn{7}{c}{ Average } & power factor per instance \\
NCC & 0.9952 & 0.9893 & 0.9886 & 0.9876 & 0.9840 \\
MILP & 0.9927 & 0.9892 & 0.9886 & 0.9876 & 0.9840 \\
\hline
\end{tabular}

\section{Reactive Power Injection}

There are two main factors influencing the reactive power injection in the power flow models: transmission lines and transformers. We measure reactive power injection by the power factors at the external grid connected to each substation. For each algorithm and each instance we obtain the smallest and average power factor among the substations and report those results in Tables IV. For example, the (NCC, $75 \%$ )-value in the upper part of Table IV is the biggest $y$ (rounded to four digits) such that the power factors at all substations in 750 out of 1000 cable layouts computed by NCC are at least $y$. The inner quantiles for both algorithms are virtually the same and the maximum values are very close. Only the overall smallest power factors for both algorithms show a notable difference, which is investigated below. In the literature, an average power factor of 0.9983 has been reported for a $17.56 \mathrm{MW}$ experimental wind farm with 24 turbines and an internal cabling at $20 \mathrm{kV}$ [18]. While a direct comparison is hardly meaningful due to different equipment, this reference suggests that cable layouts from NCC and MILP show sensible power factors.

The NCC layout with the overall smallest power factor revealed two connected substations and an exceptionally long cable $(7.3 \mathrm{~km}$, i. e., minimum turbine distance times 10.4$)$ to
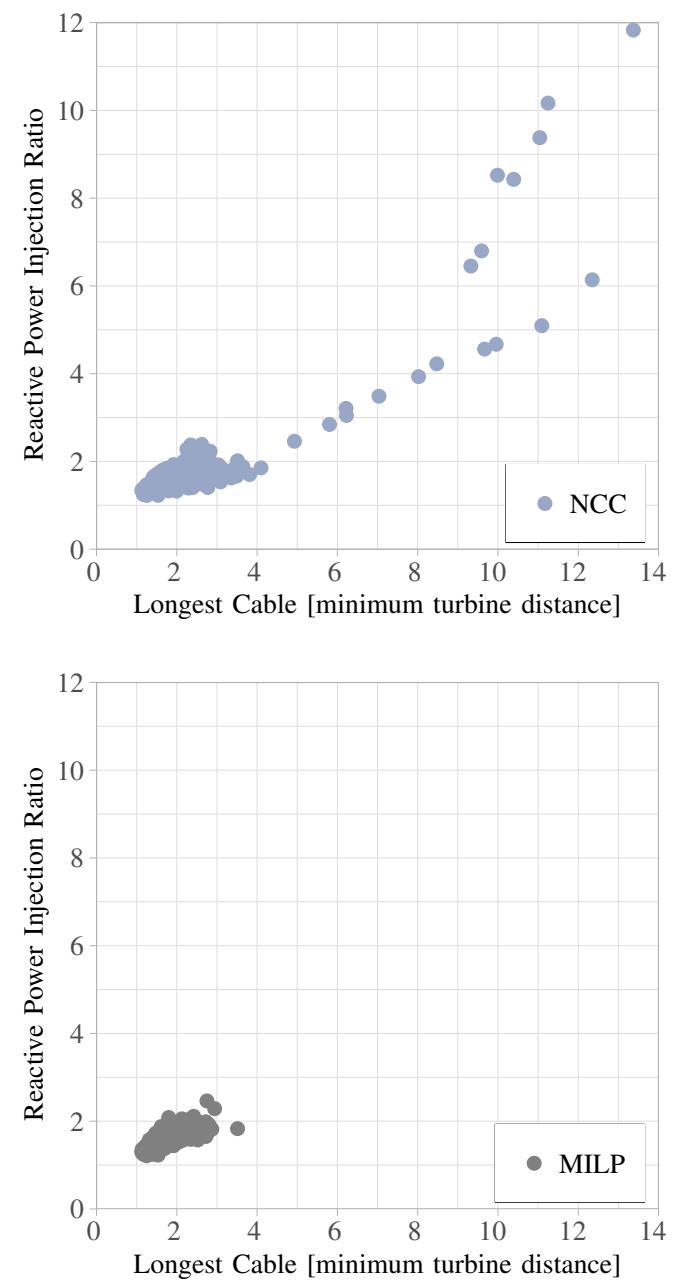

Fig. 5. Comparison of NCC and MILP cable layouts with respect to maximum cable length and highest amount of reactive power injected on transmission lines. The points (one per instance) show the length of the longest installed cable type (in multiples of the minimum turbine distance) on the abscissa and, on the ordinate, the ratio of the highest amount of reactive power injected on any line to the average amount of reactive power injection over all lines.

the substation with the smallest power factor. The simulation shows that on this line a disproportionate amount of reactive power $(356.4 \mathrm{kvar})$ is injected into the system, whereas the average reactive power injection on all transmission lines of this wind farm is 42.29 kvar. With this observation in mind, we computed this ratio of maximum absolute reactive power injection and average absolute reactive power injection over all transmission lines for all cable layouts and both algorithms.

For each algorithm, Fig. 5 shows 1000 points one for each cable layout. The abscissa of a point is the length of the longest cable in that layout and the ordinate represents the aforementioned ratio. For all algorithms we see a strong concentration of cable layouts with a length factor of at most 4 and a reactive power ratio of at most 2.5. However, for the NCC algorithm we observe several outliers with a high cable length factor and a high reactive power injection on at least one transmission line. We verified on a small sample that both values originate from 
the same transmission line. This was expected since reactive power injection increases as line length increases. We cannot explain the two apparent tendencies within the outliers. However, to keep reactive power injection low, algorithm engineers should aim for avoiding excessively long connections. This coincides with the intuition that transmission lines in wind farms connect close-by turbines.

\section{Voltage Stability}

For an investigation of voltage stability in the cable layouts, we consider the deviation of voltage levels from their nominal values in the corresponding power flow model. We use a perunit-measurement; the unit is the nominal voltage level at each bus. For the LV buses in the turbines and the HV buses in the substations we expect a measurement of $1 \mathrm{p}$. $\mathrm{u}$. since they are generator and slack buses, respectively. Our expectation was verified by the simulations. Across all instances, the lowest voltages are $0.9946 \mathrm{p}$. u. for NCC and $0.9957 \mathrm{p}$. u. for MILP. The highest voltages are $1.019 \mathrm{p} . \mathrm{u}$. for NCC and $1.011 \mathrm{p} . \mathrm{u}$. for MILP. Those values are sufficiently close to $1 \mathrm{p}$. $\mathrm{u}$. that no negative impact to the complete system is to be expected after a meaningful grid is attached to the substations.

\section{LESSONS LEARNED FOR ALGORITHM ENGINEERS}

In Sec. III we have evaluated cable layouts from the $\mathrm{NCC}$ algorithm and the MILP with respect to characteristics obtained from power flow simulations in eASiMOV. Investigating the maximum line loadings showed that cycles and connected substations can result in overloads since the split of outgoing power along two edges at a turbine need not coincide with the split of network flow as it was computed by the algorithms. An evaluation of the length of installed cables and reactive power injection showed that in some NCC cable layouts have exceptionally long cables. Not unexpectedly, those instances also showed a high reactive power injection on at least one transmission line. Algorithm engineers should therefore find ways to avoid excessively long cables.

\section{CONCLUSION}

We have presented a workflow and a corresponding application in Python 3 to analyse the output of graph algorithms for the Wind Farm Cable Layout Problem under electrical aspects. The application converts GraphML files with the algorithm output to power flow models that are imported into and simulated by the software framework eASiMOV utilizing MATPOWER. We have presented methodology to infer suitable characteristics for power system components from abstract input parameters to the graph algorithms. The NCC algorithm and the MILP approach use a network flow model for the Wind Farm Cable Layout Problem and optimize cable layouts purely with respect to the installation costs. An evaluation of those cost-optimized cable layouts with respect to line loading, active power losses, reactive power injection, and voltage stability revealed that a vast majority of cable layouts perform reasonably well with an underlying power flow instead of a network flow. For the few remaining layouts possible reasons for their poorer performance were identified so that advice to algorithm engineers for improvements of their work can be given. The presented work flow and software are sufficiently generic to be applied in other settings, e.g., with different file formats or with entirely different cable optimization problems.

\section{REFERENCES}

[1] The European Economic and Social Committee and the Committee of the Regions, "An EU strategy to harness the potential of offshore renewable energy for a climate neutral future," The European Commision, Tech. Rep., November 2020, [Accessed 28-112020]. [Online]. Available: https://eur-lex.europa.eu/legal-content/EN/ TXT/?uri=COM:2020:741:FIN

[2] P.-E. Morthorst and S. Awerbuch, "The economics of wind energy," The European Wind Energy Association, Tech. Rep., March 2009, [Accessed 30-11-2020]. [Online]. Available: http://www.ewea.org/fileadmin/files/ library/publications/reports/Economics_of_Wind_Energy.pdf

[3] G. Quinonez-Varela, G. Ault, O. Anaya-Lara, and J. R. Mcdonald, "Electrical collector system options for large offshore wind farms," IET Renewable Power Generation, vol. 1, no. 2, pp. 107-114, July 2007.

[4] S. Lumbreras and A. Ramos, "Offshore wind farm electrical design: a review," Wind Energy, vol. 16, no. 3, pp. 459-473, April 2013.

[5] C. Berzan, K. Veeramachanenil, J. McDermott, and U.-M. O'Reilly, "Algorithms for cable network design on large-scale wind farms," Massachusetts Institute of Technology, Tech. Rep., 2011, [Accessed 30-10-2020]. [Online]. Available: https://alfagroup.csail.mit.edu/sites/ default/files/documents/msrp_techreport.pdf

[6] S. Gritzbach, T. Ueckerdt, D. Wagner, F. Wegner, and M. Wolf, "Towards negative cycle canceling in wind farm cable layout optimization," Energy Informatics, vol. 1, no. 1, p. 51, October 2018.

[7] _ "Engineering negative cycle canceling for wind farm cabling," in 27th Annual European Symposium on Algorithms (ESA 2019), ser. Leibniz International Proceedings in Informatics, M. A. Bender, O. Svensson, and G. Herman, Eds., vol. 144, September 2019, pp. 55:1-55:16.

[8] S. Gritzbach, D. Wagner, and M. Wolf, "Negative cycle canceling with neighborhood heuristics for the wind farm cabling problem," in Proceedings of the Eleventh ACM International Conference on Future Energy Systems, ser. e-Energy '20. New York, NY, USA: Association for Computing Machinery, June 2020, pp. 299-307.

[9] S. Lehmann, I. Rutter, D. Wagner, and F. Wegner, "A simulatedannealing-based approach for wind farm cabling," in Proceedings of the Eighth International Conference on Future Energy Systems, ser. e-Energy '17. New York, NY, USA: Association for Computing Machinery, May 2017, p. 203-215.

[10] L. Gurobi Optimization, "Gurobi optimizer reference manual," 2021. [Online]. Available: http://www.gurobi.com

[11] U. Brandes, M. Eiglsperger, I. Herman, M. Himsolt, and M. S. Marshall, "Graphml progress report structural layer proposal," in Graph Drawing, P. Mutzel, M. Jünger, and S. Leipert, Eds. Berlin, Heidelberg: Springer Berlin Heidelberg, February 2002, pp. 501-512.

[12] M. Kyesswa, H. Çakmak, U. Kühnapfel, and V. Hagenmeyer, "A Matlab-based dynamic simulation module for power system transients analysis in the eASiMOV framework," in 2017 European Modelling Symposium (EMS), 2017, pp. 157-162.

[13] H. Çakmak, M. Kyesswa, U. Kühnapfel, and V. Hagenmeyer, "eASiMOV - a new framework for power grid analysis," in 7th Annual Conference of the KIT Energy Center, June 2018.

[14] R. D. Zimmerman, C. E. Murillo-Sánchez, and R. J. Thomas, "Matpower: Steady-state operations, planning, and analysis tools for power systems research and education,' IEEE Transactions on Power Systems, vol. 26 , no. 1 , pp. 12-19, February 2011.

[15] "Submarine power cables," Nexans, Tech. Rep., May 2008, [Accessed 6-11-2020]. [Online]. Available: https://www.nexans.de/Germany/2010/ NEX_SubmPowCables_mai08_1.pdf

[16] DIgSILENT PowerFactory User manual Version 2016, DIgSILENT $\mathrm{GmbH}$, Gomaringen, Germany, March 2016.

[17] I. Arrambide, P. M. García, J. Ugartemendia, and I. Zubia, "Evaluation of electrical losses in MVAC collector systems in offshore wind farms," Renewable Energy \& Power Quality, no. 15, pp. 287-292, April 2017.

[18] E. Diaz-Dorado, C. Carrillo, J. Cidras, and E. Albo, "Estimation of energy losses in a wind park," in 9th International Conference on Electrical Power Quality and Utilisation, November 2007, pp. 1-6. 
Karlsruher Institut für Technologie

\section{Repository KITopen}

Dies ist ein Postprint/begutachtetes Manuskript.

Empfohlene Zitierung:

Gritzbach, S.; Cakmak, H.; Mehnert, P.; Ueckerdt, T.; Hagenmeyer, V.

An evaluation of graph algorithms for the wind farm cable layout problem under electrical aspects.

2021. 56th International Universities Power Engineering Conference (UPEC): 31 Aug.-3 Sept. 2021, online, Institute of Electrical and Electronics Engineers (IEEE). $\underline{10.5445 / \mathrm{RR} / 1000139285}$

Zitierung der Originalveröffentlichung:

Gritzbach, S.; Cakmak, H.; Mehnert, P.; Ueckerdt, T.; Hagenmeyer, V.

An evaluation of graph algorithms for the wind farm cable layout problem under electrical aspects.

2021. 56th International Universities Power Engineering Conference (UPEC): 31 Aug.-3 Sept. 2021, online, Institute of Electrical and Electronics Engineers (IEEE). doi:10.1109/UPEC50034.2021.9548251 